\title{
Microcomputers in social psychophysiological research: An overview
}

\author{
LOUIS G. TASSINARY, BEVERLY S. MARSHALL-GOODELL, and JOHN T. CACIOPPO \\ University of lowa, lowa City, lowa
}

\begin{abstract}
Microcomputer systems have become commonplace in the psychophysiological laboratory during the past 5 years and are currently used in all phases of data acquisition, experimental control, and data analysis. In the past year, however, advances in microprocessor technology and scientific software have greatly extended the capabilities of these desk-top systems. Small laboratories now can afford an integrated laboratory microcomputer system and both the high-fidelity data acquisition hardware and the sophisticated analysis capabilities traditionally found in large minicomputers. We briefly describe the demands that social psychophysiological research can place on computer systems, the system presently employed in our laboratory, and a system being installed to overcome limitations on sampling rate, sampling periods, and waveform analysis.
\end{abstract}

Research and theory in social psychology have been based in large part on self-report data to assess the efficacy of the experimental manipulations, the effects of these manipulations on verbal and overt behavior, and the occurrence of postulated intervening processes. In part to avoid the limitations inherent in relying on verbal measures, there has been a growing interest among social psychologists in the potential of chronometric and noninvasive psychophysiological measures to test and refine theories of social interaction and behavior. The purpose of this paper is to briefly review the tasks typically faced in social psychophysiological research and the advances in microcomputers as they relate to observing, measuring, and quantifying social phenomena; to outline the advantages and limitations of employing inexpensive personal computers in this area of research; and to indicate the general issues involved in selecting a microcomputer system with which to automate laboratory functions. ${ }^{1}$

\section{GENERAL ISSUES}

Bridging the gap between social psychological concepts (e.g., arousal, interpersonal attraction, and emotion) and the physiological activity subserving social behavior typically requires the collection of multiple response measures in the context of multifactor experimental designs. One reason for this is that physiological systems are responsive to a wide variety of stimuli. For example, a given level of electrodermal activity (EDA) could result from the presentation of a loud noise, a deep breath, emotional imagery, or a run up a flight of stairs. In addition, the mapping from theoretically derived psychological processes into particular bodily responses often involves

Preparation of this manuscript was supported by National Science Foundation Grants BNS-8444909 and BNS-8414853. Address correspondence to L. G. Tassinary, Department of Psychology, University of Iowa, Iowa City, IA 52242. several physiological components. Social psychophysiological research is thus characterized by the analysis of intricate stimulus-response patterns.

As the instrumentation, recording techniques, and signal conditioning procedures in psychophysiology become increasingly sophisticated and standardized, ${ }^{2}$ reproducible analog (e.g., chart-and-pen) records of subtle physiological responses to a stimulus become more readily attainable. However, the specifics of particular digitization and parameter extraction processes can have dramatic effects on the fidelity of the data used for subsequent analysis (for further discussion of these issues, see Cacioppo, Petty, \& Marshall-Goodell, 1985; Glaser \& Ruchkin, 1976 , chaps. $1 \& 2$ ). To begin with, the demands placed on digitizing recording equipment vary with the response system under investigation. For example, raw surface electromyographic (EMG) signals cover roughly a 1,000fold range in both the frequency $(1-1000 \mathrm{~Hz}$ ) and amplitude (1-1,000 $\mu$ volts) domains. These characteristics require that a true high-fidelity analog-to-digital (A/D) recording system should be capable of accurately sustaining a sampling rate of at least $2 \mathrm{kHz}$ per channel and providing approximately 12-bit resolution. EDA, by contrast, covers a 20,000-fold range in the amplitude $(.005-100 \mu \mathrm{S})$ domain, yet at most only a 100 -fold range in frequency (.1-10 Hz). Thus, although the recording of EDA places minimal demands on the sampling rate of an $A / D$ converter, accurate digital recording requires some method of retaining 16 bits of resolution in the amplitude domain. ${ }^{3}$

In addition, the parameters extracted from these signals can have profound effects on the inferences drawn in an experimental investigation. Prior to the introduction of laboratory computers, quantification of EMG responses involved either counting the number of peaks in the EMG signal or calculating the mean amplitude of the integrated EMG response from a randomly selected subset of the amplitudes (cf. Lippold, 1967). The responses depicted in Figure 1, however, are not readily 

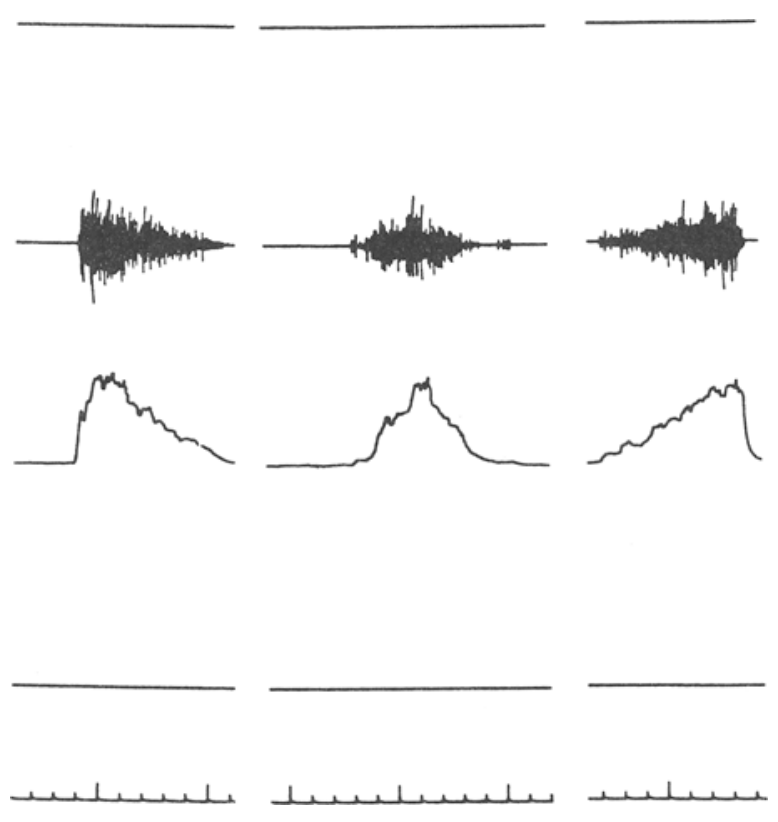

Figure 1. Raw and integrated surface EMG activity from three different contractions of the superficial forearm flexors of the right arm.

distinguishable by these methods even though they are clearly different responses (cf. Cacioppo, MarshallGoodell, \& Dorfman, 1983).

\section{RECENT DEVELOPMENTS}

It is not surprising, given the insensitive observational and quantification procedures that existed prior to the introduction of laboratory computers in social psychophysiology, that most successful early studies in this area used excessive experimental manipulations (e.g., slapping subjects across the face-R. C. Moore, 1917), and the results usually revealed general changes in physiological activity (see Ax, 1953, for an exception). Consequently, the concept of "general arousal" in social psychology overshadowed the theoretically compatible concept that subtle variations in physiological activity (e.g., visually imperceptible changes in facial efference) reflect changes in social psychological processes. The widespread availability of inexpensive microcomputers, however, has facilitated the isolation, quantification, and examination of subtle and transient responses, complex waveforms, and patterns of activity across physiological subsystems (cf. Cacioppo \& Petty, 1983; Waid, 1984). As a result, contemporary social psychophysiological research is more likely to utilize computer-intensive procedures to investigate social phenomena. For example, current research tools include various magnitude and temporal parameters of facial muscle activity. These tools have been used to distinguish spontaneous from deliberate emotional expressions (e.g., Cacioppo \& Bush, 1985), as well as to assess the effects of cooperation/competi- tion on empathetic and counterempathetic processes (Englis, Vaughan, \& Lanzetta, 1982).

\section{APPLE II/FIRST SYSTEM}

We presently employ an Apple II/FIRST microcomputer system (Scandrett \& Gormezano, 1980) interfaced to a polygraph. The addition of a $20-\mathrm{MB}$ hard disk distinguishes this system from a second-generation system described by Marshall-Goodell, Gormezano, Scandrett, and Cacioppo (1981), and places the current system somewhere between a second- and third-generation microcomputer system. Briefly, the previously described secondgeneration system consisted of an Apple II + microcomputer with $48 \mathrm{~KB}$ of random access memory (RAM), a 16-KB extended memory card, a monochrome video monitor, two Apple II minifloppy disk drives, a dot matrix printer, and a FIRST language card containing a floatingpoint coprocessor, a programmable counter/timer, an 8channel (differential) 8-bit $\mathrm{A} / \mathrm{D}$ converter, and 24 programmable digital I/O lines. An 8-channel Grass Model 7D Polygraph is used for signal conditioning prior to A/D conversion. This system was used to amplify, filter, and integrate analog measures of physiological activity from a single human subject, including up to 6 channels of EMG or electroencephalographic (EEG) activity, a single channel of EDA, and a single channel of cardiovascular activity (i.e., heart rate).

The floppy-disk based Apple II/FIRST system was able to control the timing and presentation of up to 12 independent experimental stimuli (both auditory and visual), digitize and record up to eight physiological (ana$\log$ ) signals, and extract multiple measures of response latency and response selection (e.g., yes/no, 0-9 rating scale), at a maximum aggregate rate of $800 \mathrm{~Hz}$ for brief intervals (less than $2.5 \mathrm{sec}$ ), or up to a maximum sample period of approximately $30 \mathrm{sec}$ at an aggregate rate of $25 \mathrm{~Hz}$ before pausing to store data. The digitized physiological data were logged sequentially on floppy disks for subsequent signal analysis, during which dependent variables such as mean amplitude and mean latency were extracted. The discrete response data and design information about the trial events were stored along with the physiological data for each trial to facilitate coding the data for subsequent statistical analysis and data plotting.

The hardware comprising the current 2.5-generation system remains essentially unchanged, except for the addition of a 20-MB hard disk interfaced to a videocassette recorder and multiplexer (for a more detailed description of a similar system, see Marshall-Goodell \& Gormezano, 1985). This hard disk allows faster and more reliable access to the large data files generated by our laboratory than did the floppy-disk-based system. The significance of this additional hardware should not be underestimated. In a typical experiment, the raw data from a single subject can result in up to $750 \mathrm{~KB}$ of information, and the complete data from each subject would require a number of floppy disks for storage. With the hard disk, the data 
from multiple subjects are readily accessible for signal analysis. Furthermore, the dependent variable files from an entire experiment can be accessed for statistical analyses. lyses. In practice, we have generally transmitted the dependent variable files for a particular experiment to the university's computer via a modem to take advantage of the more sophisticated statistical packages (e.g., SPSS and SAS) available on the mainframe.

The tape backup system provides a stable and compact mass storage system for programs and data on standard high-quality videocassette tapes (VHS). For example, a single videocassette can store almost $30 \mathrm{MB}$ of information, or the equivalent of about 225 floppy disks. Once backed up onto the videotape, a single record equivalent in size to a floppy disk can be located within $10 \mathrm{~min}$ and then restored to the hard disk in less than $30 \mathrm{sec}$. The multiplexer permits up to eight Apple computers to share programs and data files stored on the hard disk. Currently the hard disk is only shared between two Apple systems: one is dedicated to stimulus control and data acquisition, and the other is used for subsequent data analysis, plotting, printing of intermediate results, and transmitting data to the mainframe for final analysis.

The FIRST software system (Scandrett \& Gormezano, 1980 ) is a flexible dictionary-based high-level language similar to FORTH (C. H. Moore, 1974), but specifically optimized for an Apple II environment which includes an AM9511 floating-point coprocessor. Numerical processing time is reduced by the use of reverse Polish notation with the argument(s) for all operators and commands taken from a numerical stack. The core of the FIRST language consists of 600 available functions called words, and programming involves defining new functions which are sequential combinations of previously defined words: The sequential nature of operations in FIRST requires a structured programming style in which the "GOTO" statements common to early versions of BASIC and FORTRAN are not permitted. This modular architecture allows any individual components of a FIRST program to be extracted and tested independently, and reused in subsequent programs.

In spite of the power, flexibility, and moderate cost of the present system, several limitations prompted us to seek a third-generation system. First, the 8-bit processor in the Apple is relatively slow when processing the large arrays of 32-bit numbers generated by our signal analysis program. "Relatively slow" in this context means that we may spend several hours extracting the first five moments from the trial-by-trial EMG waveforms, and another hour computing second-by-second heartrate data from a single subject. Furthermore, this system is unable to process numbers with more than 32-bit precision during intermediate calculations (see Cacioppo, Marshall-Goodell, \& Dorfman, 1983, for a description of some of these analytic procedures).

Second, the maximum of $64 \mathrm{~KB}$ of RAM available in an Apple II placed severe restrictions on the time over which physiological signals can be rapidly sampled and stored without pausing to write to the disk, even given the continued use of low resolution 8-bit A/D convertors (see Simon, 1973, for a brief discussion of the "continuous data problem'") ${ }^{4}$; and it effectively prevented the storage of unintegrated EMG signals. In addition, many of the data analysis programs had to be subdivided into smaller intermediate programs, each of which fits within the available memory. This subdivision introduced a complex series of steps in the data analysis process which are employed in almost all studies but which require user intervention at each intermediate step. Third, the graphic capabilities of the Apple II/FIRST system are restricted by the Apple's low-resolution monochrome display $(160 \times 280$ pixels $)$ and the lack of sophisticated graphics routines in the standard lexicon of FIRST.

Finally, although FIRST is extremely flexible, it remains a "procedure-oriented" language (Considine, 1976) much like BASIC and FORTRAN. Essentially, it provides a syntax which determines how the process of solving a particular problem is to be carried out. As a result, it has only rudimentary built-in functions, and consequently more complex operations must be developed essentially from the ground up. This can become a nontrivial problem at every stage of data acquisition and analysis since complex experimental designs and analyses are not always easily parsed into the existing lexicon. It is this lack of built-in analytic and display functions that prevented us from doing any real-time data analysis or display, limited our utilization of graphics, and led us to resort to transmitting data to the university mainframe for anything more than rudimentary statistical analyses. This is not to imply that such functions cannot be developed in FIRST, but rather to say simply that they have not been developed and that to do so would require a substantial investment of time and effort for developing, testing, and optimizing such functions.

\section{IBM PCAT/ASYST SYSTEM}

The IBM PCAT ("Advanced Technology") is rapidly becoming the standard by which all third-generation microcomputers are judged (Finefrock, Hale, \& Aarons, 1985). Built around the 16/24-bit Intel 80286 microprocessor, the AT architecture can support up to $16 \mathrm{MB}$ of RAM while featuring integrated memory management for multitasking support. This support provides up to one GB of virtual address space per task and includes intertask communication, synchronization, memory sharing, and task scheduling. In addition, up to four internal storage devices (a maximum of two hard disk drives and two diskette drives) are supported along with 16 levels of system interrupts and six direct memory access (DMA) channels. The system is characterized by an open-bus structure that allows multiple microprocessors to share the system's resources, including memory.

Our current developing system is based on two IBM PCATs running under PC DOS. One machine is dedicated to laboratory control and data acquisition, and the 
other is dedicated to data reduction, data analysis, and manuscript preparation. Each machine has $3 \mathrm{MB}$ of RAM, an Intel 80287 coprocessor running at $5 \mathrm{MHz}$, a 33-MB hard disk drive, a 60-MB streaming cartridge tape drive, a 1.2-MB floppy disk drive, an Enhanced Graphics Adaptor card, a color graphics display terminal, two serial ports, one parallel port, and a game port. In addition, the laboratory system has four data acquisition and control cards located in an expansion chassis which provide two 1-MHz clocks, six independent 16-bit counters, 32 bits of programmable digital I/O, four 12-bit D/A channels, and 16 differential 12-bit A/D channels. The particular A/D cards we use allow an aggregate sampling rate of up to $50 \mathrm{kHz}$ using DMA transfers to memory.

Although a communication network will eventually be used to integrate this laboratory system, the fact that each microcomputer has its own tape drive makes this upgrade less urgent. It is now possible to backup easily the data from single or multiple subjects onto the tape from the laboratory computer, and then restore the data onto the hard disk of the other computer. While this could require up to an hour using the videotape backup system, the streaming tape drives are much faster, more flexible, and more reliable, allowing individual file access from anywhere on the tape within 2 or $3 \mathrm{~min}$.

Although FIRST has proven to be a powerful laboratory software system within the confines of the Apple II environment, it has limitations and was not designed to take advantage of the enhanced features in the more powerful IBM environment. However, a scientific software package named ASYST, which was specifically designed for the IBM PC series (Hultquist, 1985), has recently been released.

ASYST, like FIRST, is a FORTH-like language which utilizes reverse Polish notation and requires that all operators and commands work on the top elements of a number stack. Unlike FIRST, however, ASYST is more accurately described as a "problem-oriented"' language: that is, it represents a language specifically designed for convenience of program development in scientific and industrial research settings (Considine, 1976). Essentially this means that the core words in the lexicon of ASYST include not only the rudimentary level of commands which comprise the base vacabulary of FIRST, such as the command to plot a pixel at a specified location in the display window (PLOT in FIRST; P! in ASYST), but also numerous high-level commands that execute complex operations such as preparing $\mathrm{X}-\mathrm{Y}$ plots and histograms (e.g., HISTOGRAM). In addition, these high-level commands assume default features that are changed easily by a set of "adjectives." For example, the command HORIZONTAL LOGARITHMIC modifies the display produced by the command XY.AUTO.PLOT so that the horizontal axis is scaled logarithmically rather than linearly. Other modifiers are available to change solid lines to dashed or dotted lines, to change plot symbols, to insert labels, and so forth. The use of modifiers with the high-level commands of ASYST for data acquisition and graphical output insulates the programmer from some of the peculiarities of specific I/O hardware, thus making ASYST programs relatively device-independent.

Not only does ASYST contain many efficient and thoroughly tested high-level commands not available in FIRST, but even the elementary arithmetic operators (i.e., $+,-, *, /,{ }^{* *}$ ) can be directly applied to entire arrays as easily as to individual scalars without the need for program loops. Each array can contain single- or double-precision entries which can be integer, real, or complex numbers (complex numbers cannot be manipulated in FIRST), and can have up to 16 dimensions. The only software limitation is that each array can contain a maximum of $64 \mathrm{~KB}$ of data which, depending on the data type used, allows between $2^{15}$ single-precision elements and $2^{13}$ double-precision complex elements per array.

In addition, entire arrays can be pushed on the number stack, with the number stack being limited to simultaneously storing up to 30 arrays and scalars. In laboratory applications which require the use of large arrays, this represents a major improvement over FIRST, where the number stack can hold up to 256 scalars, but each element of an array requires a separate entry in the stack at any given point in time during program execution. New number stacks of any desired size can be created in ASYST as needed and an array editor is also available to facilitate examination and manipulation of subarrays. ${ }^{5}$

Four modules of ASYST are currently available. Module 1 (SYSTEM/GRAPHICS/STATISTICS) contains the basic language which includes arithmetic operations and trigometric functions; descriptive statistical functions including the mean, variance, mode, and higher moments; high-level graphics functions; a full-screen text editor; an on-line HELP facility; and data I/O routines. Module 2 (ANALYSIS) contains a number of single command analysis routines including the Fast Fourier Transform (FFT), integration, differentiation, smoothing, peak detection, least squares approximations, and both polynomial and matrix arithmetic. Module 3 (DATA ACQUISITION) contains data acquisition templates, both digital and ana$\log$, for six commerically available laboratory interfaces (e.g., Cyborg, Data Translation, IBM, Keithley, Metrabyte, and Tecmar), as well as provides the facility for customized interfacing to other hardware systems. Softwarecontrolled data acquisition can be performed in "background" while analysis is performed in "foreground" if the sampling rate permits, but very fast sampling rates are obtainable using ASYST since both DMA and interrupt processing are fully supported. Module 4 (GPIB/IEEE-448) allows ASYST to control and sample from any laboratory device that has an GPIB/IEEE-448 data bus.

Rudimentary testing indicates that previous limitations in sampling rate, RAM, real-time graphics, and programming have been greatly reduced. With the affordable computational power available to most social psychophysio- 
logical laboratories through the use of third-generation microcomputers, the insights of Charles Wrigley nearly 30 years ago finally speak to the individual investigator (Wrigley, 1957):

[The psychologist's] computational load will be considerably lightened, exact methods can be used where approximations have had to suffice, less effort need be given to the development of short cuts, and more time will be available for his principal work, the formulation and testing of hypotheses. (p. 508)

\section{REFERENCES}

Ax, A. F. (1953). The physiological differentiation between fear and anger in humans. Psychosomatic Medicine, 15, 433-442.

Basmajian, J. V., Clifford, H. C., Mcleod, W. D., \& Nunnally, H. N. (1975). Computers in electromyography. London: Butterworth.

CACIOPPO, J. T., \& Bush, L. K. (1985). The effects of voluntary control over facial expressions on facial EMG responses. Psychophysiology, 18, 585-586. (Abstract)

Cacioppo, J. T., Marshall-Goodell, B., \& Dorfman, D. (1983). Skeletomuscular patterning: Topographical analysis of the integrated electromyogram. Psychophysiology, 20, 269-283.

Cacioppo, J. T., Marshall-Goodell, B., \& Gormezano, I. (1983) Social psychophysiology: Bioelectrical measurement, experimental control, and analog-to-digital data acquisition. In J. T. Cacioppo \& R. E. Petty (Eds.), Social psychophysiology: A sourcebook. New York: Guilford Press.

CAClopPo, J. T., \& PetTy, R. E. (EDS.). (1983). Social psychophysiology: A sourcebook. New York: Guilford Press.

Cacioppo, J. T., Petty, R. E., \& Marshall-Goodell, B. (1985), Physical, social, and inferential elements of psychophysiological measurement. In P. Karoly (Ed.), Measurement Strategies in Health Psychology: Vol. 1. (pp. 263-300). New York: Wiley \& Sons.

Coles, M. G. H., Donchin, E., \& PoRges, S. (Eds.) (in press). Handbook of Psychophysiology. New York: Guilford Press.

Considine, D. M. (ED.). (1976). Van Nostrand's scientific encyclopedia (5th ed.). New York: Van Nostrand Reinhold.

Englis, B., Vaughan, K. B., \& Lanzetra, J. T. (1982). Conditioning of counter-empathetic emotional responses. Journal of Experimental Social Psychology, 8, 375-391.

Finefrock, M. C., Hale, B. C., \& Aarons, D. (1985). The enhanced AT: A personal vision. PC Magazine, 4(13), 120-137.

Glaser, E. M. \& RuchkIN, D. S. (1976). Principles of neurobiological signal analysis. New York: Academic Press.

Guld, C., Rosenfalck, A., \& Willison, R. G. (1970). Technical factors in recording electrical activity of muscle and nerve. Electroencephalography \& Clinical Neurophysiology, 28, 399-413.

HultQuist, P. F. (1985). Minipower for the PC. PC: The Independent Guide to IBM Personal Computers, 4, 186-197.

LIPPOLD, O. C. J. (1967). Electromyography. In P. H. Venables and I. Martin (Eds.), A manual of psychophysiological methods. Amsterdam: North Holland.

Marshall-Goodell, B., Gormezano, I., Scandrett, J., \& Cacioppo, J. T. (1981). The microcomputer in social psychophysiological research: An Apple II/FIRST laboratory. Sociological Methods \& Research, 9, 502-512.

MARShall-Goodell, B., \& Gormezano, I. (1985). Apple II/FIRSTCorvus networks. Behavior Research Methods, Instruments, \& Computers, 17, 168-172.

Martin, I., \& Venables, P. H. (EDs.). (1980). Techniques in psychophysiology. Chichester: Wiley \& Sons.
McHugo, G., \& Lanzetta, J. T. (1983). Methodological decisions in social psychophysiology. In J. T. Cacioppo \& R. E. Petty (Eds.), Social Psychophysiology: A sourcebook (pp. 630-665). New York: Guilford Press.

MoORE, C. H. (1974). FORTH: A new way to program minicomputers. Journal of Astronomy \& Astrophysics Supplement, 15, 497-511.

MOORE, R. C. (1917). Laboratory tests of anger, fear, and sex instincts. American Journal of Psychology, 28, 390-395.

RugG, M. D., Fletcher, R. P., \& LyKKen, D. T. (1980). Computers in psychophysiological research. In I. Martin \& P. H. Venables (Eds.), Techniques in psychophysiology (pp. 583-595). Chichester: Wiley \& Sons.

SCANDRETT, J., \& GoRmezano, I. (1980). Microprocessor control and A/D data acquisition in classical conditioning. Behavior Research Methods \& Instrumentation, 12, 120-125.

SimoN, W. (1973). Advanced techniques for the small computer. In B. Weiss (Ed.), Digital computers in the behavioral laboratory. New York: Appleton-Century-Crofts.

Stern, R. M., RAY, W. J., \& DAvis, C. M. (1980). Psychophysiological recording. New York: Oxford University Press.

STRODT, P. A. (1984). Microcomputer methods for social scientists. Beverly Hills: Sage.

WAID, W. (1984). Sociophysiology. New York: Springer-Verlag.

WRIGLEY, C. (1957). Electronic computers in psychological research. American Psychologist, 12, 501-508.

\section{NOTES}

1. Our discussion of these issues will be necessarily brief. Interested readers might wish also to consult Basmajian, Clifford, McLeod, and Nunnally (1975), Cacioppo, Marshall-Goodell, and Gormezano (1983), McHugo and Lanzetta (1983), Rugg, Fletcher, and Lykken (1980), and Strodt (1984).

2. Stern, Ray, and Davis (1980) provide a good introduction to the physiological basis of these measures, their bioelectrical characteristics, and appropriate signal-conditioning procedures. For more advanced discussions of signal-conditioning procedures and standards, see Coles, Donchin, and Porges (in press), Guld, Rosenfalck, and Willison (1970), and Martin and Venables (1980).

3. It is possible to utilize special purpose hardware to extract specific parameters of the measured signals (e.g., peak amplitude) and store only these parameters for analysis. However, there are a number of limitations to this research strategy. First, this strategy presumes that the investigator can specify, a priori, the relevant and irrelevant parameters of the physiological signals. Second, not only may this strategy actually misrepresent the actual psychological response (e.g., as did the practice of recording one physiological measure to assess "arousal"), it also needlessly restricts exploratory data analysis and discourages or prevents subsequent reanalysis of the data when new theoretical or empirical developments suggest alternative parameters of interest. Given the recent cost-effective technological advances in data acquisition, processing speed, and compact mass storage devices, these limitations are no longer necessary. Hence, the benchmarks presented and the discussion of microcomputer applications in social psychophysiological research are based on the presupposition that unanalyzed signal is being recorded and digitally stored for later use.

4. High-speed ( $\geq 25 \mathrm{~K}$ samples per second), high-resolution (12- or 16-bit) A/D converters are available for the Apple II, but the limited RAM severely constrains real-time multiple channel recording with such fidelity to only a few seconds.

5. Both FIRST and ASYST create compact files which are binary representations of the data, but ASYST can also read and write standard PC-DOS comma-delimited ASCII files. This facility permits ASYST to easily create data files which can be subsequently analyzed by standard statistical and database management software and allows ASYST programs to operate on existing data files from other sources. 